\title{
Contributions to dietary protein intake in a British adult population
}

\author{
L. K. M. Bain ${ }^{1}$, P. K. Myint ${ }^{2}$, A. Jennings ${ }^{1}$, M. A. H. Lentjes ${ }^{3}$, A. A. Mulligan ${ }^{3}$, R. N. Luben ${ }^{3}$, \\ K. T. Khaw ${ }^{3}$ and A. A. Welch ${ }^{1}$ \\ ${ }^{1}$ Norwich Medical School, University of East Anglia, Norwich, NR4 7TJ, ${ }^{2}$ Aberdeen Gerontological and \\ Epidemiological INterdisciplinary Research Group (AGEING), Epidemiology Group, Institute of Applied Health \\ Sciences, School of Medicine \& Dentistry, Aberdeen, AB25 2ZN and ${ }^{3}$ Department of Public Health and Primary Care, \\ Institute of Public Health, University of Cambridge, Cambridge, CBI 8RN
}

Protein, a key nutrient for health, has a number of significant roles in the body including being a component of skeletal muscle, and so is integral in the prevention of sarcopenia ${ }^{(1,2)}$. However, there is limited knowledge of the contribution of different types of protein (namely animal and plant based) which have been associated with other diseases such as Type II diabetes ${ }^{(3)}$. The purpose of this study was to investigate sources of protein as there are currently no recommendations regarding the source or quality of protein to be consumed, and little previous research has investigated the contributing sources to the UK diet.

We analysed a random representative sub-cohort of the European Prospective Investigation into Cancer and Nutrition - Norfolk (EPIC-Norfolk cohort of 25,000 men and women) comprised of 4443 men and women aged 39-80. We aimed to identify the contributing sources to total dietary protein \%en (percentage of energy) from four aggregated variables of protein intake derived from 7-day food diaries, by quintiles of intake. These categories were animal-land (meat and meat products), animal-marine (fish, seafood), animal-derived (e.g. milk, cheese, eggs) and plant (all protein from plant sources). The average protein intake $\%$ en was $15.0 \%$ and $15 \cdot 8 \%$ for men and women, respectively. Total animal protein intake accounted for a mean of $10 \cdot 1 \%$ and $10 \cdot 8 \%$ of energy intake for men and women respectively, whilst plant protein accounted for $4.7 \%$ of energy for both men and women. All analyses were conducted in Stata, version 11.0.
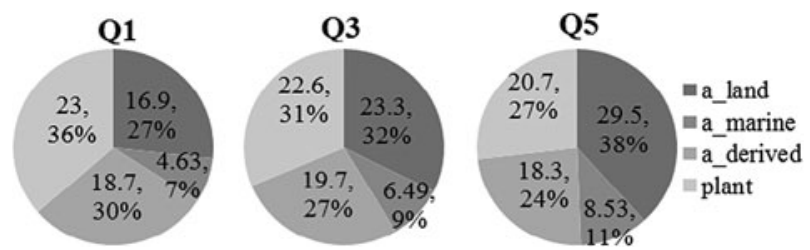

Fig. 1. Amount of protein source (animal-land, -marine, -derived, and plant) in $\mathrm{g} / \mathrm{d}$, and percentage contribution to total protein intake by quintiles of total protein \%en.

The contribution of protein sources differed across quintiles of total protein \%en. Increases in land and marine based protein $(\mathrm{P}$ trend $<0.001)$ and decreases in plant and animal derived intakes were seen with increasing total protein $\%$ en $(\mathrm{P}$ trend $<0.001$ and $0 \cdot 03$, respectively).

In conclusion protein intake for both men and women in this study was predominantly from animal sources. Further investigation of the sources of protein and differences in quality of associated nutrients may be important for sarcopenia and other noncommunicable chronic diseases.

1. Gibney M. J. et al. Nutrition and metabolism. 1st ed. Ames, Iowa: Iowa State Press, 2003.

2. Welch A. A. Nutritional influences on age-related skeletal muscle loss. Proc Nutr Soc 2014;73(1):16-33.

3. van Nielen M. et al. Dietary protein intake and incidence of type 2 diabetes in Europe: the EPIC-InterAct Case-Cohort Study. Diabetes Care 2014;37(7):1854-62. 